\title{
Non-tuberculosis Mycobacterium Tenosynovitis with Rice Bodies in a Patient with Systemic Lupus Erythematosus
}

\author{
Yuichiro Fujieda ${ }^{1}$, Keita Ninagawa ${ }^{1}$, Yuichiro Matsui ${ }^{2}$, Michihiro Kono ${ }^{3}$, \\ Tamotsu Kamishima ${ }^{4}$, Norimasa Iwasaki ${ }^{2}$ and Tatsuya Atsumi ${ }^{1}$
}

\begin{abstract}
:
Infectious disease with various presentations in systemic lupus erythematosus often resembles lupus flare. A 37-year-old woman presented with a swollen left index finger that had not resolved, despite 7 years of immunosuppressive treatment. MRI showed rice-body formation in the flexor tendon sheath and tenosynovectomy demonstrated chronic synovitis with epithelioid granuloma. A mycobacterial culture confirmed invasive mycobacterial tenosynovitis due to Mycobacterium chelonae. The patient was treated with moxifloxacin and clarithromycin and completely recovered.
\end{abstract}

Key words: tenosynovitis, non-tuberculosis mycobacterium, systemic lupus erythematosus, Mycobacterium chelonae, rice-body formation

(Intern Med 59: 2317-2320, 2020)

(DOI: 10.2169/internalmedicine.4671-20)

\section{Introduction}

Systemic lupus erythematosus (SLE) is an autoimmune disease with diverse clinical manifestations that is characterized by remission and flares. Arthritis is one of the most common disease manifestations in SLE. Infectious arthritis in SLE often resembles a disease flare and hence is difficult to diagnose. Rice-body formations, which could be detected by imaging modalities such as magnetic resonance imaging (MRI), have been reported in non-tuberculosis mycobacterium $(\mathrm{NTM})$ tenosynovitis $(1,2)$. We herein report a case of refractory arthritis in an SLE patient, who was diagnosed with NTM tenosynovitis after the detection of rice bodies on MRI and the confirmation of Mycobacterium chelonae on a lower temperature tissue culture. The patient was successfully treated with moxifloxacin and clarithromycin.

\section{Case Report}

A 37-year-old Japanese woman with a 25-year history of SLE, presented with swelling of the proximal interphalangeal (PIP) joint of the left index finger, which had been present since she was 30 years of age. She has been treated with prednisolone (PSL; $15 \mathrm{mg}$ ) and mizoribine (MZB; 150 $\mathrm{mg}$ ) in recent years. The first manifestations were butterfly rash, arthritis, lupus nephritis and leukopenia, and she was treated with steroid pulse therapy and cyclophosphamide. The swelling of the PIP joint initially improved with immunosuppressive therapy at 33 years of age. However, it relapsed with hypocomplementemia and an elevated anti-DNA antibody titer at 35 years of age. After the addition of tacrolimus, the patient's laboratory data showed improvement, but the joint swelling remained unchanged. A radiograph of the left index finger was normal, with the exception of swelling. Ultrasonography of the left index finger revealed hypoechoic soft-tissue masses along the flexor tendon

\footnotetext{
${ }^{1}$ Department of Rheumatology, Endocrinology and Nephrology, Faculty of Medicine and Graduate School of Medicine, Hokkaido University, Japan, ${ }^{2}$ Department of Orthopaedic Surgery, Faculty of Medicine and Graduate School of Medicine, Hokkaido University, Japan, ${ }^{3} 3$ rd Department of Internal Medicine, Hokkaido P.W.F.A.C Obihiro-Kosei General Hospital, Japan and ${ }^{4}$ Faculty of Health Sciences, Hokkaido University, Japan Received: February 16, 2020; Accepted: May 1, 2020; Advance Publication by J-STAGE: June 15, 2020 Correspondence to Dr. Yuichiro Fujieda, edaichi@med.hokudai.ac.jp
} 


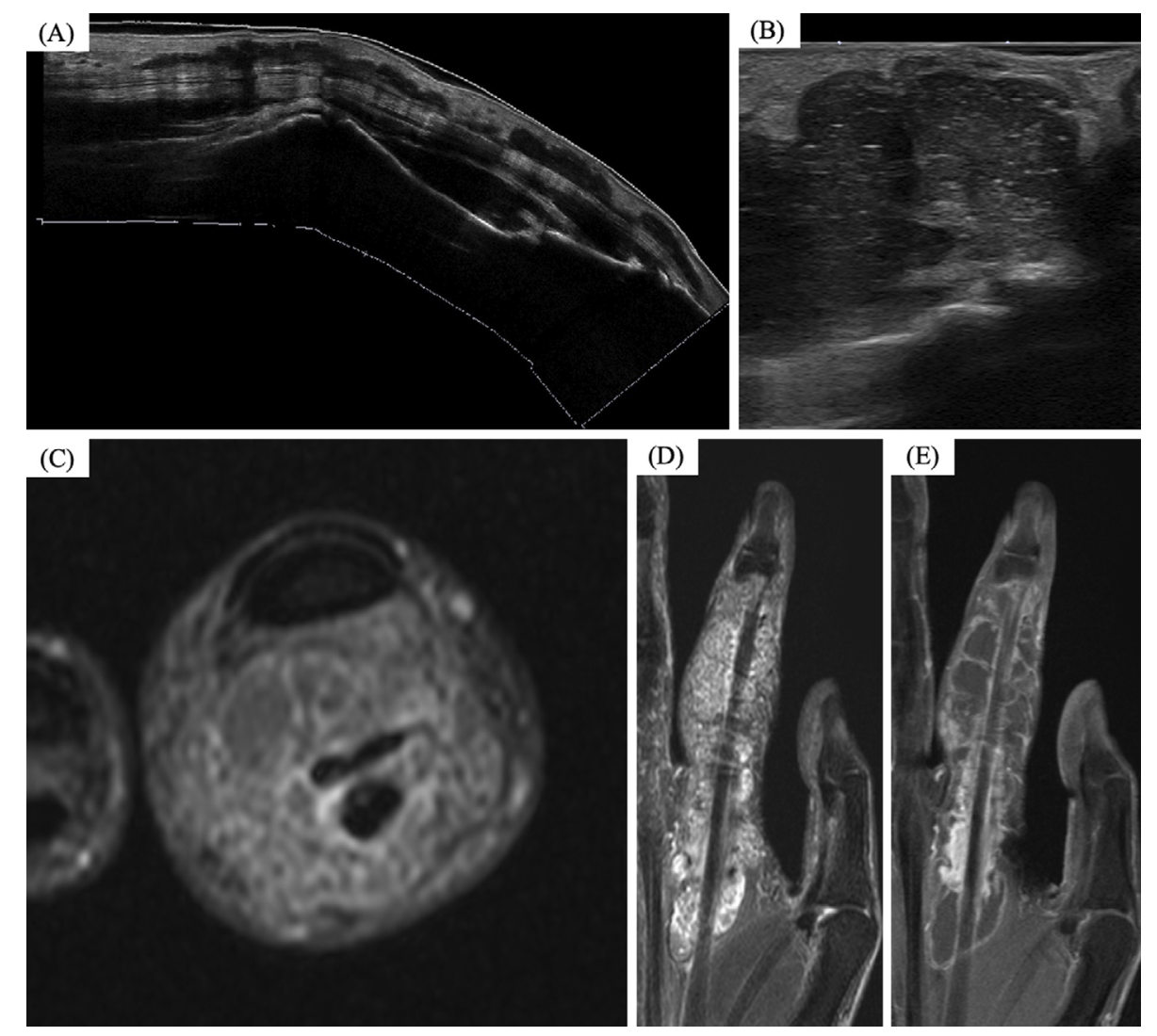

Figure 1. A and B: Ultrasonography of the left index finger. Hypoechoic soft-tissue masses along the flexor tendon (A) with multiple internal hyperechoic 'flecks' (B). C, D and E: Fluid-sensitive magnetic resonance imaging of the left index finger. Numerous tiny nodules of low signal intensity ( $C$ and $D$ ), which have no contrast enhancement effect (E). Post-contrast enhancement of the flexor tendon sheath and thickened synovium adjacent to the flexor tendon is demonstrated (E).

(Fig. 1A) with multiple internal hyperechoic 'flecks' (Fig. 1B). Fluid-sensitive MRI of the left index finger revealed numerous tiny nodules of low signal intensity (Fig. 1C, D), which had no contrast enhancement effect (Fig. 1E). These findings were compatible with rice body formation in the flexor tendon sheath. Post-contrast enhancement of the flexor tendon sheath and thickened synovium adjacent to the flexor tendon was demonstrated (Fig. 1E). There were no findings of NTM infection in other sites. Synovectomy was performed and rice-body formation was found (Fig. 2A, B). The histopathological examination of the mass lesion demonstrated chronic synovitis complicated by epithelioid granuloma (Fig. 2C) with increased numbers of fibroblast-like synoviocytes (Fig. 2D). Acid-fast staining was negative. However, a colony was detected on a mycobacterial culture incubated at $28-32^{\circ} \mathrm{C}$. M. chelonae was identified by matrix-assisted laser desorption/ionization timeof-flight mass spectrometry (MALDI-TOF MS) and genome sequencing, pointing to a diagnosis of tenosynovitis of the hand due to $M$. chelonae. A drug sensitivity test was performed using the microdilution method as follows: Imipenem/Cilastatin: S (MIC=4.0), Clarithromycin: S (MIC= 0.12), Minocycline: R (MIC>8.0), Levofloxacin: $\mathrm{R}$ ( $\mathrm{MIC}=$ 4.0). Moxifloxacin and clarithromycin were administered for 6 months. The swelling disappeared, and the remission has been maintained.

\section{Discussion}

NTM is a ubiquitous environmental organism found in water and animals, that causes chronic infection in humans (3). A previous report indicated that approximately 7\% of patients with NTM infection were immunocompromised, including autoimmune disease patients treated with immunosuppressive drugs (4). Further, a majority of these infections occurred after trauma (5). In a meta-analysis, all patients presented with swelling and pain, and $74 \%$ of all cases of hand tenosynovitis involved only one finger (4). Although we need to be aware that the epidemiology of NTM infection varies due to regional differences, a literature review described that the most common organisms of NTM in hand infection is $M$. marinum (82\%), followed by $M$. chelonae (5\%), M. kansasii (3\%), and M. intracellulare (3\%) (4). M. chelonae is a rapidly growing mycobacteria (RGM) belonging to Group 4 of the Runyon classification (6). M. chelonae has been reported in association with hand infection more than other RGMs, such as M. abscessus (7). As acid-fast staining is usually negative in these infections, special ZiehlNeelsen staining and incubation at $28-32^{\circ} \mathrm{C}$ in LowensteinJensen medium must be performed (5). M. chelonae is iden- 


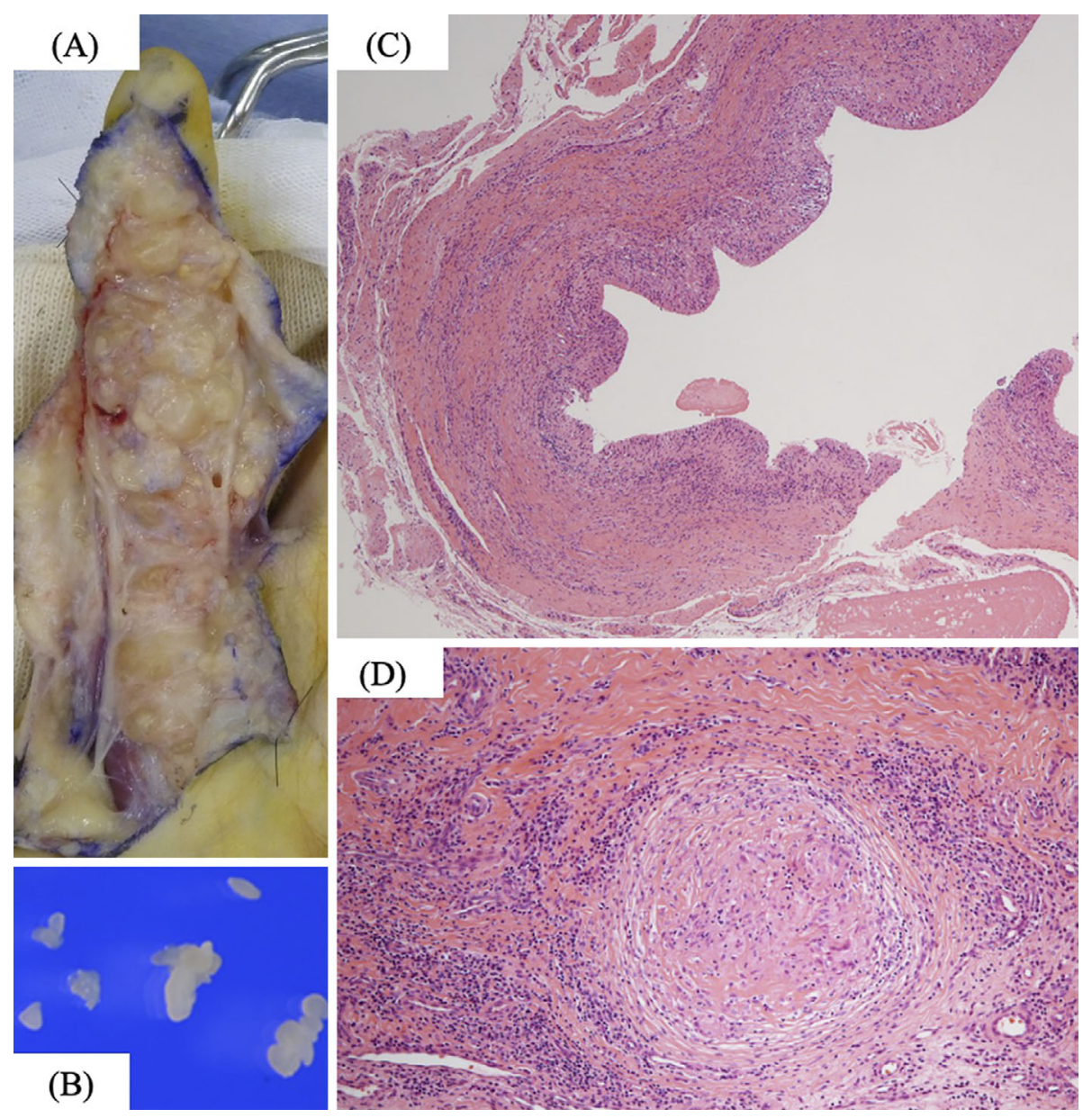

Figure 2. A: Numerous rice bodies were found during surgery. B: A specimen including rice bodies was collected by synovectomy. $C$ and D: Hematoxylin and Eosin staining of a synovial biopsy specimen shows epithelioid granuloma and increasing fibroblast-like synoviocytes $(\mathrm{C}, \times 4$ magnification, and $\mathrm{D}, \times \mathbf{1 0}$ magnification).

tified by several methods, including real-time polymerase chain reaction, DNA-DNA hybridization, MALDI-TOF MS, and genome sequencing (8).

Early surgery and appropriate culture guided antibiotic therapy are crucial in the management of NTM hand tenosynovitis. A combination antibiotic therapy is recommended. The use of amikacin, ciprofloxacin, and clarithromycin was reported in previous studies. Clarithromycin was reported to be the most active agent in inhibiting the growth of $M$. chelonae (9). The duration of treatment should be at least 6 months, but should be no longer than 12 months (4).

A cohort study reported that NTM infection occurred in $1.5 \%$ of SLE patients (10). In a review of the relevant literature on NTM infections in SLE patients (11), M. chelonae (26.8\%) was the most common, followed by $M$. kansasii (21.4\%) and $M$. avium complex (14.3\%). Extra-pulmonary lesions are more common than tuberculosis infection. Skin and soft tissue infection (55.4\%) are the most common locations, followed by the joints (23.2\%), and lung (21.4\%) (11). However, the frequency of NTM species and the prevalence of infection sites may be different in Japan. Bone marrow infection, which causes pancytopenia has also been reported (12). These manifestations resemble a disease flare. Thus, when the immunosuppressive treatment is ineffective, NTM infection should be considered as a differential diagnosis.

Rice bodies caused in tenosynovitis are reported in rheumatoid arthritis, tuberculosis, and NTM infection (1). The pathogenesis of rice body formation remains unclear, but experts have suggested that they arise from microinfarcted synovium or that the early rice body appears de novo and causes progressive enlargement by fibrin deposition (13). MRI is considered the imaging modality of choice for rice bodies, which appear iso- or slightly hyperintense in comparison to skeletal muscle on T1- and T2-weighted imaging (1). In our case, the MRI findings were not typical and required further diagnostic steps.

Based on our experience, we encourage clinicians to suspect NTM infection when patients with SLE present refractory mono-arthritis that is resistant to immunosuppressive treatment. Rice bodies detected on MRI assisted our diagnosis and lower temperature tissue culturing was needed to confirm the diagnosis of $M$. chelonae, which is a common NTM infection in SLE patients.

The authors state that they have no Conflict of Interest (COI). 
Yuichiro Fujieda and Keita Ninagawa contributed equally to this work.

\section{References}

1. Yamamoto D, Tada K, Suganuma S, Ikeda K, Tsuchiya H. Nontuberculous Mycobacterium or fungus induced chronic tenosynovitis with rice body of the hand. J Hand Surg Asian Pac Vol 22: 337-342, 2017.

2. Hoffman KL, Bergman AG, Hoffman DK, Harris DP. Tuberculous tenosynovitis of the flexor tendons of the wrist: MR imaging with pathologic correlation. Skeletal Radiol 25: 186-188, 1996.

3. Lee EY, Ip JW, Fung BK, Ted UE. Mycobacterium chelonae hand infection: a review. Hand Surg 14: 7-13, 2009.

4. Balague N, Uckay I, Vostrel P, Hinrikson H, Van Aaken I, Beaulieu JY. Non-tuberculous mycobacterial infections of the hand. Chir Main 34: 18-23, 2015.

5. Nguyen A, Ipaktchi K, Livermore M, Banegas R. Mycobacterium chelonae infection of the hand presenting as a collar-button abscess. Hand Surg 20: 149-151, 2015.

6. Wallace RJ Jr. Recent changes in taxonomy and disease manifestations of the rapidly growing mycobacteria. Eur J Clin Microbiol Infect Dis 13: 953-960, 1994.

7. Chen HY, Chen CY, Huang CT, et al. Skin and soft-tissue infection caused by non-tuberculous mycobacteria in Taiwan, 1997 2008. Epidemiol Infect 139: 121-129, 2011.
8. Tortoli E. Microbiological features and clinical relevance of new species of the genus Mycobacterium. Clin Microbiol Rev 27: 727752, 2014.

9. Brown BA, Wallace RJ Jr, Onyi GO, De Rosas V, Wallace RJ 3rd. Activities of four macrolides, including clarithromycin, against Mycobacterium fortuitum, Mycobacterium chelonae, and $\mathrm{M}$. chelonae-like organisms. Antimicrob Agents Chemother 36: 180184, 1992.

10. Mok MY, Wong SS, Chan TM, Fong DY, Wong WS, Lau CS. Non-tuberculous mycobacterial infection in patients with systemic lupus erythematosus. Rheumatology (Oxford) 46: 280-284, 2007.

11. Shaharir SS, Sahari NS, Fuad ZM, et al. Non-tuberculous mycobacterium bacteraemia in a pregnant systemic lupus erythematosus (SLE) patient: a case review and pooled case analysis. Clin Rheumatol 37: 837-847, 2018.

12. Yazisiz V, Erbasan F, Inan D, et al. Bone marrow infection caused by Mycobacterium avium complex in a patient with systemic lupus erythematosus. Lupus 19: 323-326, 2010.

13. Jeong YM, Cho HY, Lee SW, Hwang YM, Kim YK. Candida septic arthritis with rice body formation: a case report and review of literature. Korean J Radiol 14: 465-469, 2013.

The Internal Medicine is an Open Access journal distributed under the Creative Commons Attribution-NonCommercial-NoDerivatives 4.0 International License. To view the details of this license, please visit (https://creativecommons.org/licenses/ by-nc-nd/4.0/).

\footnotetext{
(C) 2020 The Japanese Society of Internal Medicine Intern Med 59: 2317-2320, 2020
} 